\title{
Influences of Desmin and Keratin 19 on Passive Biomechanical Properties of Mouse Skeletal Muscle
}

\author{
Sameer B. Shah, ${ }^{1,2}$ James M. Love, ${ }^{1}$ Andrea O’Neill, ${ }^{3}$ Richard M. Lovering, ${ }^{4}$ \\ and Robert J. Bloch ${ }^{3}$ \\ ${ }^{1}$ Fischell Department of Bioengineering, University of Maryland, College Park, MD 20742, USA \\ ${ }^{2}$ Departments of Orthopaedic Surgery and Bioengineering, University of California, San Diego, La Jolla, CA 92093, USA \\ ${ }^{3}$ Department of Physiology, University of Maryland School of Medicine, Baltimore, MD 21201, USA \\ ${ }^{4}$ Department of Orthopaedics, University of Maryland School of Medicine, Baltimore, MD 21201, USA
}

Correspondence should be addressed to Sameer B. Shah, sbshah@ucsd.edu

Received 28 May 2011; Accepted 10 September 2011

Academic Editor: J.-P. Jin

Copyright (C) 2012 Sameer B. Shah et al. This is an open access article distributed under the Creative Commons Attribution License, which permits unrestricted use, distribution, and reproduction in any medium, provided the original work is properly cited.

\begin{abstract}
In skeletal muscle fibers, forces must be transmitted between the plasma membrane and the intracellular contractile lattice, and within this lattice between adjacent myofibrils. Based on their prevalence, biomechanical properties and localization, desmin and keratin intermediate filaments (IFs) are likely to participate in structural connectivity and force transmission. We examined the passive load-bearing response of single fibers from the extensor digitorum longus (EDL) muscles of young ( 3 months) and aged (10 months) wild-type, desmin-null, K19-null, and desmin/K19 double-null mice. Though fibers are more compliant in all mutant genotypes compared to wild-type, the structural response of each genotype is distinct, suggesting multiple mechanisms by which desmin and keratin influence the biomechanical properties of myofibers. This work provides additional insight into the influences of IFs on structure-function relationships in skeletal muscle. It may also have implications for understanding the progression of desminopathies and other IF-related myopathies.
\end{abstract}

\section{Introduction}

Lateral force transmission, both across the sarcolemma and within a single myofiber, is believed to be an important component of the biomechanical function of muscle [14]. Forces must be transmitted both between the plasma membrane and the intracellular myofibrillar lattice, and also within this lattice, from myofibril to myofibril. Based on their prevalence, biomechanical properties and localization, intermediate filaments (IFs) are strong candidates to regulate such lateral transmission. IFs composed of desmin and keratins 8 and 19 have been identified as key components of mature skeletal muscle [5-7]. Considerable evidence links these IFs to roles in structural connectivity and mechanical function.

Desmin, a type III IF expressed exclusively in striated muscle, localizes around Z-disks in the extramyofibrillar space, around mitochondria, at the periphery of cell nuclei, and at costameres, which are periodic sarcolemmal protein complexes lying over the Z-disks and M-bands of peripheral myofibrils [8-14]. Structurally, the loss of desmin results in misaligned myofibrils and disrupted costameres $[15,16]$. Mechanically, muscles lacking desmin display reduced maximal isometric force, increased fatigability, poor transverse and longitudinal coupling, and slight increases in passive stiffness, particularly in older animals [17-19]. Although there are discrepancies regarding the susceptibility of desmin-null muscles to injury, these may be attributable to differences in the strains of desmin-null mice probed, the identity of muscles tested, or injury protocols imposed $[17,18]$.

Keratin 19 (K19) and keratin 8 (K8), type I and type II IF proteins, respectively, are the best characterized of the keratins expressed in mature striated muscle. At the sarcolemma, K8 and K19 colocalize with and specifically interact with costameric proteins, including dystrophin, 
overlying both the Z-disks and M-bands of adjacent myofibrils $[20,21]$. Within the myofibrillar lattice, K19 and K8 concentrate primarily around Z-disks [21]. Structurally, the absence of K19 results in the partial disruption of costameres and the dramatic separation of the sarcolemma from adjacent myofibrils by a large gap within which mitochondria accumulate $[18,22]$. A small but significant shift in the lateral spacing between myofibrils is also observed in the absence of K19; however, myofibrillar desmin localization is undisturbed $[18,22]$. Physiologically, the absence of K19 results in decreased maximal isometric force and increased plasma creatine kinase levels $[18,22]$. Double-null muscles, lacking both K19 and desmin, display increased susceptibility to injury and significant increases in membrane instability. Decreases in specific tension are greater than that of K19-null muscles, but equal to those in desmin-null muscles [18].

Given the possibility that extracellular matrix remodeling could influence the mechanical properties of whole muscle [23-25], experiments on single fibers have been performed to examine structural connectivity during passive mechanical stretch $[26,27]$. Analysis of desmin-null fibers confirmed the necessity of desmin for myofibrillar and nuclear alignment during mechanical loading. Unlike observations in whole muscle, however, isolated desmin-null fibers do not appear to display misaligned costameres, even in the presence of considerable shear force. In addition, desmin-null fibers are more compliant than their wild-type counterparts.

In this study, we have extended the examination of biomechanical roles for IFs in single fibers. We investigated the structural changes and tensile load-bearing response of passively loaded fibers harvested from the extensor digitorum longus (EDL) muscles of young (3 months) and middle-aged (10 months) wild-type, desmin-null, K19-null, and desmin/K19 double-null FVB mice. We observed that, although fibers are more compliant in both desmin and K19null knockout mice, the structural changes that occur within the two types of fibers during loading are different. Our data suggest that IFs influence force transmission in myofibers through multiple biomechanical pathways.

\section{Methods}

2.1. Animals. Female FVB mice homozygous for the desminnull (des -/-), K19-null (K19 -/-), and desmin/K19 double-null genotypes were bred and genotyped as described previously [18] and compared to age- and sex-matched wild-type FVB mice (Taconic, Hudson, NY, USA). Mice were studied at two ages, 3 months and 10 months. They were euthanized by carbon dioxide inhalation immediately prior to tissue harvest. Institutional Animal Care and Usage (IACUC) Committees of the University of Maryland School of Medicine and the University of Maryland, College Park, approved all animal use protocols.

\subsection{Fiber Dissection and Attachment to Mechanical Apparatus.} Tissue harvest, muscle storage, and fiber dissection protocols have been described previously [27]. Briefly, EDL muscles were dissected from mouse hindlimbs, bathed in relaxing solution for $60 \mathrm{~min}$ and placed in a glycerol-based storage solution for a minimum of $24 \mathrm{~h}$ and a maximum of one week at $-20^{\circ} \mathrm{C}$. All solutions were made in the presence of the protease inhibitor leupeptin. Segments of single intact fibers were carefully dissected and transferred in relaxing solution to a chamber housed in a custom-made mechanical apparatus. This apparatus is similar to that previously used $[26,27]$, with the only difference being the modification of the structural frame and stage insert to be mounted on an inverted wide-field fluorescence microscope (Nikon TE-2000PFS, Melville, NY, USA). Within the chamber, the segment was securely tied with 10-0 monofilament suture to pins projecting from a force transducer (Aurora Scientific 405A, Aurora, Ontario, Canada) and a rotational bearing (Newport MTRS, Irvine, Calif, USA). This configuration enabled the high-resolution imaging of fiber and sarcomere geometry during passive loading. To minimize any experimental artifacts associated with glycerol-mediated skinning of fibers during storage, segments displaying abnormal discoloration, localized swelling, or otherwise irregular geometry or appearance were discarded.

\subsection{Passive Mechanical Protocol and Imaging. The fiber} segment was brought to its resting length, determined as the knot-to-knot segment length at which passive tension was just measurable above the noise level of the force transducer. The segment was preconditioned by three loading cycles of $15 \%$ fiber strain, resulting in a change in resting length of $<3 \%$ from the resting length before preconditioning. The segment was then loaded in increments of $\sim 10 \%$ of the segment length at a rate of $0.05 \mathrm{~mm} / \mathrm{s}$. At each length, after two minutes of stress relaxation, images of the fiber were captured in a middle region of the fiber, and steady-state force (within $5 \%$ of the final force value) was recorded. During the $2 \mathrm{~min}$ of stress relaxation, the imaging field was adjusted to visualize the same region and focal plane of the fiber at each strain. Phase-contrast imaging was performed on an inverted microscope (Nikon TE-2000PFS, Melville, $\mathrm{NY}$ ), using 10x and 20x CFI Plan Apo objectives (Nikon). Exposure times were 5-20 ms; image acquisition was controlled by Elements software (Nikon, Melville, NY, USA). A custom built environmental chamber (Precision Plastics, Beltsville, $\mathrm{Md}$, USA) maintained temperature $\left(25^{\circ} \mathrm{C}\right)$ and humidity during imaging.

2.4. Calculation of Biomechanical Parameters. Image analysis was performed using Elements software and Micron software (Westover Scientific, Mill Creek, Wash, USA). Fiber crosssectional area was calculated using a circular approximation, based on diameters measured from a phase contrast image of the fiber at each strain. Area was multiplied by fiber length to estimate fiber volume, assuming a cylindrical fiber. Cauchy (true) stress $\left(\sigma_{C}\right)$ was calculated by dividing force by the current cross-sectional area at each strain, and LaGrangian stress $\left(\sigma_{\mathrm{LG}}\right)$ was calculated by dividing force by the crosssectional area of the undeformed fiber. Fiber strain $\left(\varepsilon_{\mathrm{F}}\right)$ was calculated as the ratio of the imposed deformation to the resting knot-to-knot fiber length. Sarcomere length (SL) 
was calculated based on the average spacing between 20 consecutive Z-disks, as measured from phase images of fibers at each fiber strain. SL in different regions of the fiber varied by less than $0.1 \mu \mathrm{m}$, and therefore SLs were measured at three locations per image and averaged. Sarcomere strain $\left(\varepsilon_{\mathrm{SL}}\right)$ was calculated as the ratio of the change in SL to the SL at resting fiber length. Strain in the sarcolemmal plasma membrane in the vicinity of the imaged sarcomeres, or local membrane strain, was calculated based on changes in the spacing between membrane markers, or pieces of residual debris on the external surface of the fiber; local membrane strain $\left(\varepsilon_{\mathrm{M}}\right)$ was calculated as the ratio of the change in spacing between membrane markers to marker spacing at resting fiber length. Radial strain $\left(\varepsilon_{\mathrm{R}}\right)$ was calculated as the ratio of the change in fiber diameter to the fiber diameter at resting fiber length. Poisson's ratio $(v)$ was calculated based on the power-law relationship between longitudinal and radial strain. Biomechanical parameters and their relationships with each other are summarized in Table 1.

2.5. Statistics. Regression analysis was performed using Excel (Microsoft, Seattle, Wash, USA). One-way ANOVAs examining the effect of genotype were performed independently on mechanical data from young and aged mice, since an additional double-null group was evaluated in aged mice. Data from double-null mice were not considered for subsequent two-way ANOVA analysis, which examined effects and interactions of age and genotype on mechanical parameters. ANOVA and post hoc two-tailed $t$-tests assuming unequal variance were performed using Prism (Graph Pad Software, Inc., La Jolla, Calif, USA). Values are given as mean \pm SEM.

\section{Results}

3.1. Intermediate Filament Influences on Passive Properties of Fibers from Aged Animals. We examined the response of EDL fibers harvested from 10-month-old wild-type, desmin-null, K19-null, and desmin/K19 double-null mice to tensile loading to examine the relative contributions of each intermediate filament to fiber passive mechanical properties. A summary and a brief description of measured and calculated parameters are provided in Table 1.

For stress-strain curves, regression coefficients were used to compare the relative compliance of fiber populations. Cauchy stress was plotted against fiber strain for each experiment and fit using quadratic regression (Figure 1(a), cf. [26]). Fibers from all genotypes displayed a strong quadratic relationship between stress and fiber strain $\left(r^{2}>0.78\right.$ for all experiments), indicating an elastic modulus that varies linearly with strain. ANOVA revealed a significant effect of genotype on the quadratic regression coefficient $(P<$ 0.0001). Post hoc comparison of the quadratic coefficients revealed that wild-type fibers were significantly stiffer than desmin-null $(P<0.002)$, K19-null $(P<0.006)$, and double-null $(P<0.002)$ fibers (Figure 1(b)). There were no significant differences in the quadratic regression coefficients of K19-null and desmin-null or desmin-null and doublenull fibers. Interestingly, however, a significant difference
$(P<0.03)$ was observed between K19-null and double-null fibers for Cauchy stress-strain curves.

To determine whether these differences in fiber populations were strictly a result of changes in fiber geometry or also inherent load-bearing ability, LaGrangian stress was plotted against fiber strain (Figure 1(c)). Use of the undeformed area to calculate stress had the effect of flattening the curves, so linear regression was used $\left(r^{2}>0.81\right.$ for all experiments) to describe the relationship between stress and fiber strain. ANOVA again revealed a significant effect of genotype on the regression coefficient $(P<0.0001)$. Comparison of linear coefficients confirmed that wild-type fibers were significantly stiffer than desmin-null $(P<0.005)$, K19-null $(P<0.007)$, and double-null $(P<0.004)$ fibers (Figure $1(\mathrm{~d}))$. There were no significant differences in the linear regression coefficients of K19-null and desmin-null, desmin-null and double-null, or K19-null and double-null fibers. Consistent with results from Cauchy stress-strain relationships, though, a strong but nonsignificant trend $(P<0.09)$ was observed for the latter.

Analysis of radial strain revealed additional differences in the mechanical response of fibers from different genotypes (Figure 1(e)). First-order exponential functions were used to describe the relationship between radial and longitudinal strains $\left(r^{2}>0.83\right.$ for all experiments). Although, as expected, ANOVA revealed a significant effect of genotype on the exponential decay constant $(P<0.04)$, post hoc comparison (Figure 1(f)) revealed that wild-type fibers exhibited tighter coupling between longitudinal and radial strains than desmin-null $(P<0.04)$, but not K19-null $(P<0.24)$ fibers. Wild-type fibers also trended towards a higher time constant (tighter coupling) than double-null fibers, but were not significantly different $(P<0.06)$. As was the case for stress-strain relationships, there was no difference between the relationship between radial and longitudinal strain for desmin-null and double-null fibers.

We further examined the coupling of longitudinal and radial strains in the context of fiber anisotropy, by calculating Poisson's ratio. A power-law was used to fit the relationship between longitudinal and radial strain, with the exponent denoting Poisson's ratio for each fiber. $R^{2}$ values for each experiment were reasonably high $\left(r^{2}>0.62\right)$, though not as strong as an exponential regression fit. For all genotypes, Poisson's ratios exceeded 0.5, indicating significant anisotropy. There were no significant differences in Poisson's ratio between any of the genotypes (Mean \pm SEM for wildtype: $0.81 \pm 0.10$; des $-/-: 0.63 \pm 0.08$; ker $-/-$ : $0.71 \pm$ 0.05 ; double KO: $0.61 \pm 0.04$ ), though a trend towards a slightly higher Poisson's ratio was observed for wild-type fibers compared to double-null fibers $(P<0.09)$.

Consistent with the high Poisson's ratios, when changes in fiber volume were plotted against longitudinal strain, each genotype displayed a decrease in volume with increasing strain, indicating that loading was not isovolumic. This decrease was usually linear, and therefore linear regression was used to fit this data $\left(r^{2}>0.68\right)$. Wild-type fibers displayed the most dramatic decrease in volume (Mean slope \pm SEM: $-0.38 \pm 0.11$ ), though there were no significant differences in first-order regression slopes between any of the genotypes (des -/-: $-0.16 \pm 0.05$; ker $-/-$ : $-0.22 \pm 0.04$; 


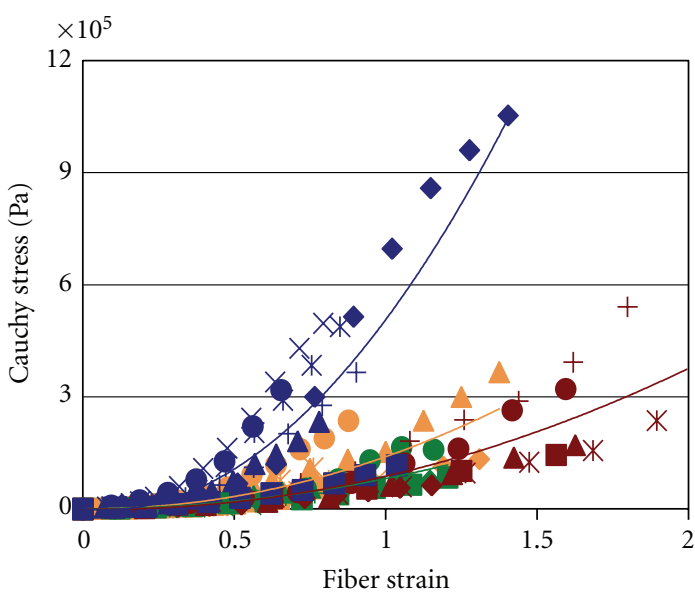

(a)

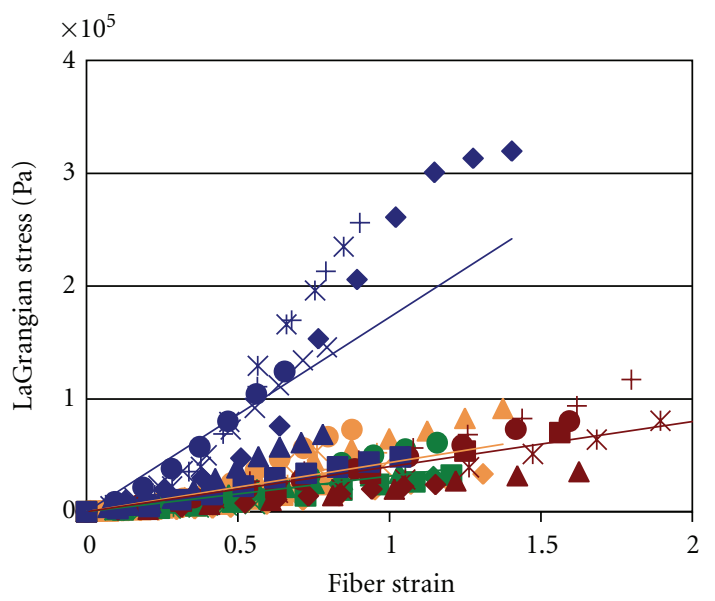

(c)

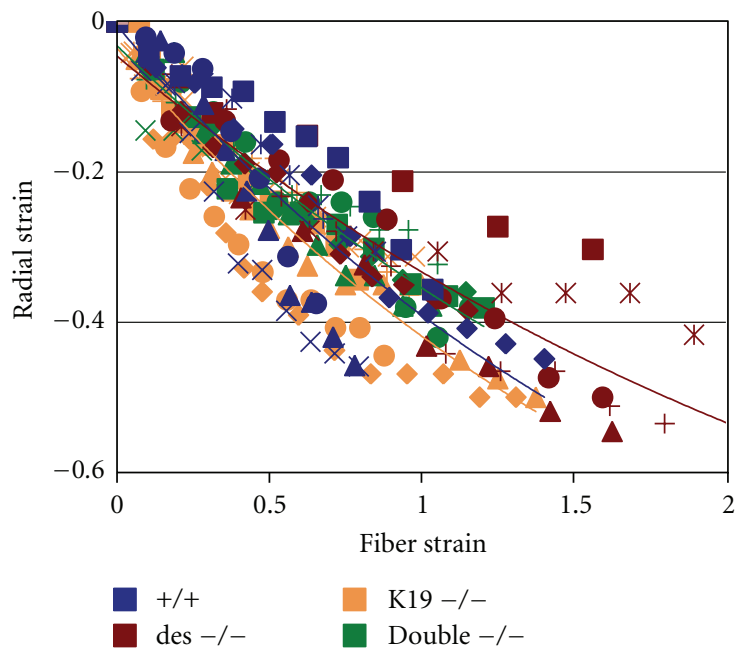

(e)

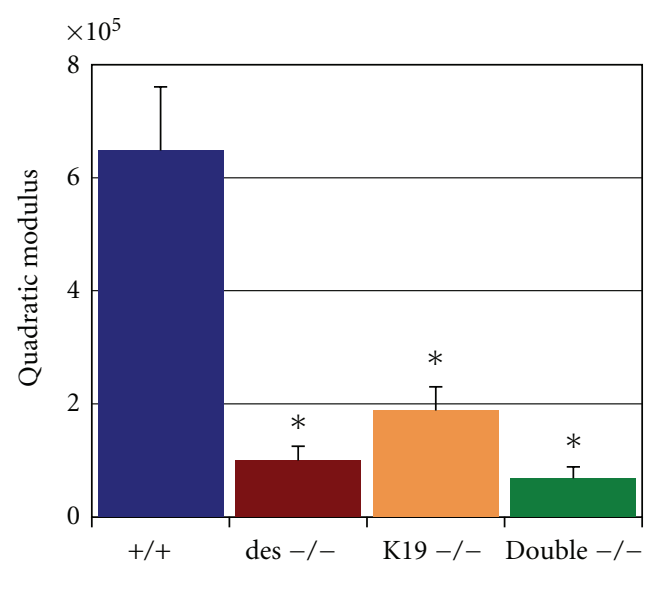

(b)

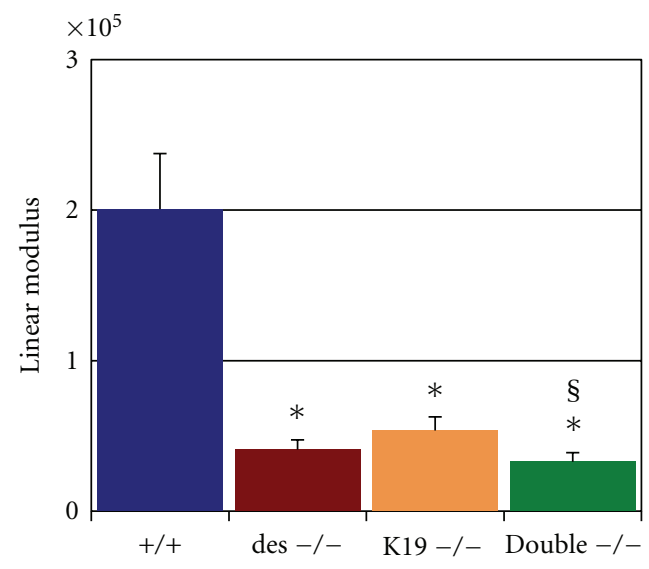

(d)

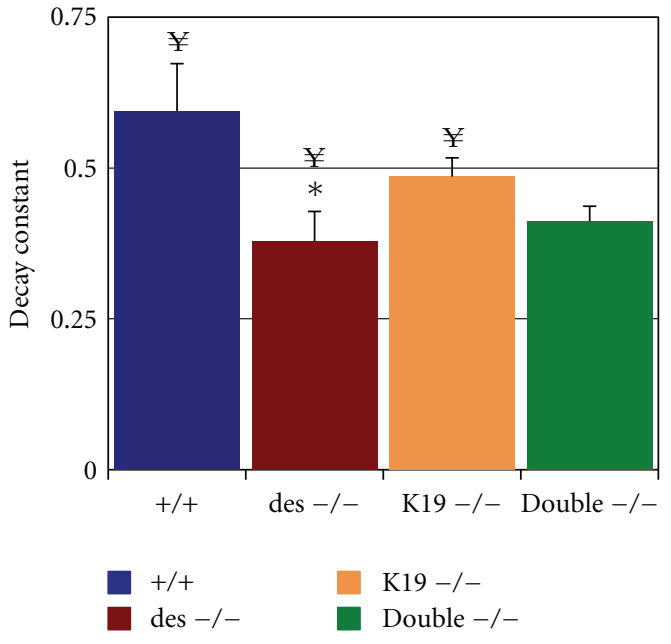

(f)

Figure 1: Mechanical response of fibers from aged animals. Raw data (a, c, e) and regression coefficients (b, d, f) for (a-b) Cauchy stress-fiber strain (quadratic regression); (c-d) LaGrangian stress-fiber strain (linear regression); (e-f) radial strain-fiber strain (exponential regression) for fibers from 10-month-old wild-type (blue), desmin-null (red), K19-null (orange), and double-null (green) animals ( $n=7$ fibers/genotype). Different symbols within a color group represent data from individual experiments. Displayed regression lines in (a), (c), and (e) represent averaged coefficients for individual experiments reported in (b), (d), and (f). * Indicates significant difference from wildtype; § indicates significant difference from K19-null; $¥$ indicates significant difference from corresponding genotype in younger animals. $P$ values are reported in the text. 
TABLE 1: Summary and explanation of biomechanical parameters.

\begin{tabular}{|c|c|c|c|}
\hline Parameter (units) & Symbol & Formula & Lay description of parameter and relevance \\
\hline Force $(\mathrm{N})$ & $F$ & - & Nonnormalized force \\
\hline Undeformed fiber length (m) & $L_{\mathrm{o}}$ & - & Initial knot-knot fiber length \\
\hline Undeformed diameter $(\mathrm{m})$ & $d_{\mathrm{o}}$ & - & Initial fiber diameter \\
\hline Undeformed area $\left(\mathrm{m}^{2}\right)$ & $A_{\mathrm{o}}$ & - & Initial fiber cross-sectional area \\
\hline Deformed fiber length (m) & $L_{f}$ & - & $\begin{array}{l}\text { Fiber length following longitudinal } \\
\text { deformation }\end{array}$ \\
\hline Deformed diameter $(\mathrm{m})$ & $d_{f}$ & - & $\begin{array}{l}\text { Fiber diameter following longitudinal } \\
\text { deformation }\end{array}$ \\
\hline Deformed area $\left(\mathrm{m}^{2}\right)$ & $A_{f}$ & - & $\begin{array}{l}\text { Fiber cross-sectional area following } \\
\text { longitudinal deformation }\end{array}$ \\
\hline $\begin{array}{l}\text { Longitudinal fiber strain } \\
\text { (dimensionless) }\end{array}$ & $\varepsilon_{\mathrm{L}}$ & $\left(L_{f}-L_{\mathrm{o}}\right) / L_{\mathrm{o}}$ & $\begin{array}{l}\text { Normalized longitudinal } \\
\text { deformation-percentage fiber extension }\end{array}$ \\
\hline Fiber volume & $V_{f}$ & $L_{f} * A_{d}$ & $\begin{array}{l}\text { Fiber volume after deformation, assuming } \\
\text { a cylindrical geometry }\end{array}$ \\
\hline Radial strain (dimensionless) & $\varepsilon_{\mathrm{R}}$ & $d_{f}-d_{\mathrm{o}} / d_{\mathrm{o}}$ & $\begin{array}{l}\text { Normalized lateral } \\
\text { deformation-percentage reduction in } \\
\text { fiber diameter following longitudinal } \\
\text { deformation; increased radial strain } \\
\text { represents stronger coupling between } \\
\text { longitudinal and lateral responses. }\end{array}$ \\
\hline Cauchy (true) stress $(\mathrm{Pa})$ & $\sigma_{C}$ & $F / A_{d}$ & $\begin{array}{l}\text { Force normalized to deformed } \\
\text { area-couples force generation to changes } \\
\text { in 3D-fiber geometry }\end{array}$ \\
\hline LaGrangian stress $(\mathrm{Pa})$ & $\sigma_{\mathrm{LG}}$ & $F / A_{\mathrm{o}}$ & $\begin{array}{l}\text { Force normalized to undeformed } \\
\text { area-measure of inherent load-bearing } \\
\text { ability, decoupled from changes in } \\
\text { 3D-fiber geometry }\end{array}$ \\
\hline $\begin{array}{l}\text { Poisson's ratio } \\
\text { (dimensionless) }\end{array}$ & $v$ & $\left(1+\varepsilon_{\mathrm{L}}\right)=\left(1+\varepsilon_{\mathrm{L}}\right)^{-\nu}$ & $\begin{array}{l}\text { Measure of relation between longitudinal } \\
\text { strain and radial strain—lateral strain } \\
\text { transmission }\end{array}$ \\
\hline
\end{tabular}

TABle 2: Summary of parameters that display significant post hoc differences $(P<0.05)$ between genotypes for fibers harvested from aged mice. Raw data, means, and standard errors are provided in Figure 1. Parameters that display non-significant trends $(P<0.09)$ are provided in parentheses. NS: No significant difference.

\begin{tabular}{llll}
\hline Genotype & des $-/-$ & $\mathrm{K} 19-/-$ & Double $-/-$ \\
\hline$+/+$ & $\sigma_{\mathrm{LG}}, \sigma_{C}, \varepsilon_{\mathrm{R}}$ & $\sigma_{\mathrm{LG}}, \sigma_{C}$ & $\sigma_{\mathrm{LG}}, \sigma_{C},\left(\varepsilon_{\mathrm{R}}\right),(\nu),\left(V_{f}\right)$ \\
des $-/-$ & & $\mathrm{NS}$ & $\mathrm{NS}$ \\
$\mathrm{K} 19-/-$ & & & $\sigma_{C},\left(\sigma_{\mathrm{LG}}\right),\left(\varepsilon_{\mathrm{R}}\right)$ \\
\hline
\end{tabular}

double KO: $-0.13 \pm 0.04)$. As for Poisson's ratio, a trend towards a slightly higher magnitude of regression slope was observed for wild-type fibers compared to double-null fibers $(P<0.08)$. Significant differences between parameters are summarized in Table 2.

3.2. Intermediate Filament Influences on Passive Properties of Fibers from Young Animals. Passive biomechanical analysis was then performed on EDL fibers harvested from 3-monthold wild-type, desmin-null, and K19-null mice, to probe whether phenotypes observed in the older 10-month-old animals originate at earlier time points. As was observed for older animals, fibers from all genotypes displayed a strong quadratic relationship between Cauchy stress and fiber strain $\left(r^{2}>0.82\right.$ for all experiments; Figure 2(a)) and a linear relationship between LaGrangian stress and fiber strain $\left(r^{2}>\right.$ 0.86 for all experiments; Figure 2(c)). For both Cauchy and LaGrangian stress-strain, ANOVA revealed a significant effect of genotype on regression coefficients (quadratic: $P<$ 0.002; linear: $P<0.0002$, resp.). Post hoc comparison of the quadratic coefficients revealed that wild-type fibers were significantly stiffer than desmin-null $(P<0.01)$ and K19null $(P<0.01)$ fibers (Figure 2(b)). Similarly, comparison of linear coefficients confirmed that wild-type fibers were significantly stiffer than desmin-null $(P<0.01)$ and K19null $(P<0.01)$ fibers (Figure $2(\mathrm{~d}))$.

Analysis of radial strain in fibers from younger animals, in contrast to observations in fibers from older animals, also did not reveal any differences between desmin-null and K19-null genotypes (Figure 2(e)). First-order exponential functions were again used to describe the relationship between radial and longitudinal strain $\left(r^{2}>0.78\right.$ for all experiments). ANOVA revealed a significant effect of genotype on the exponential decay constant $(P<0.0003)$. Post hoc comparison of the time constants revealed that wild-type fibers exhibited a stronger inverse relationship, and 


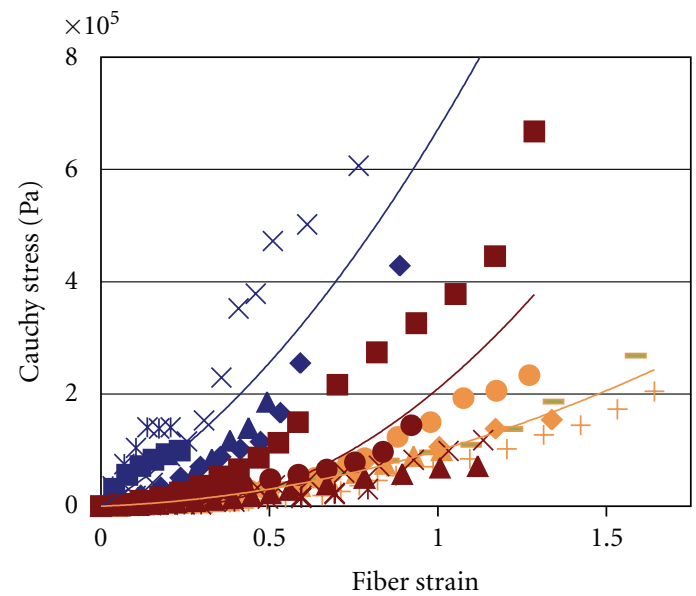

(a)

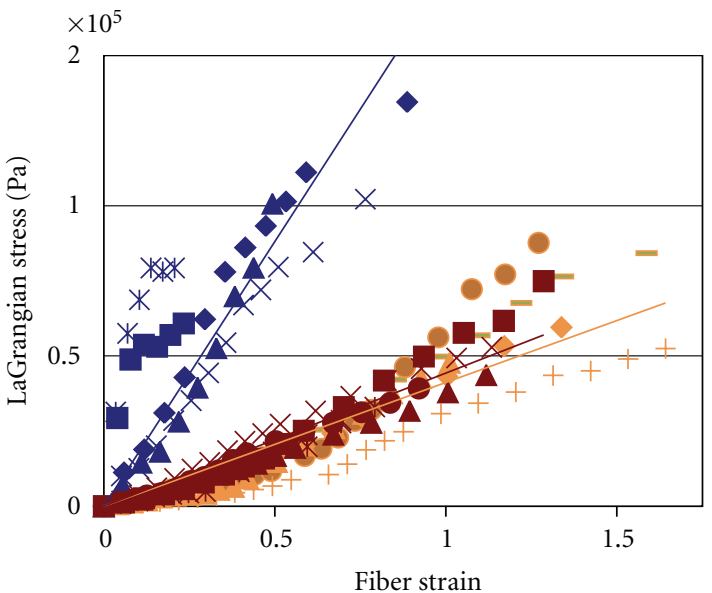

(c)

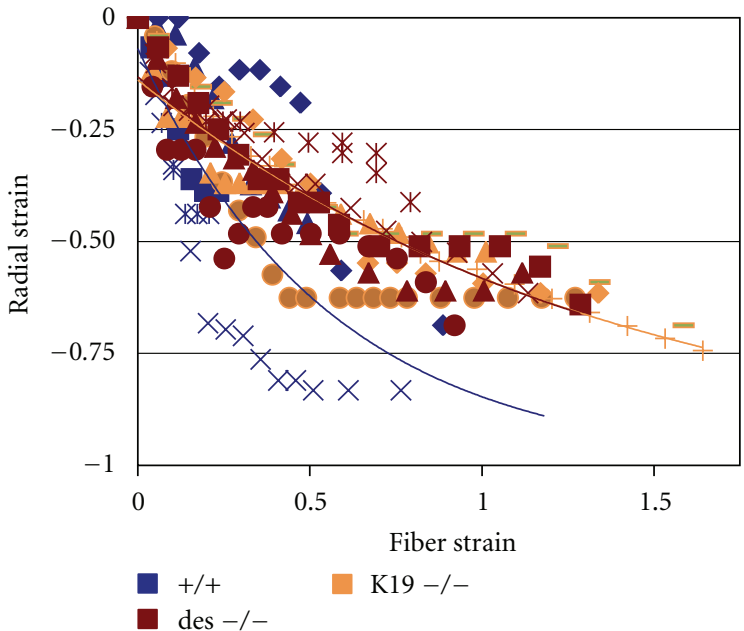

(e)

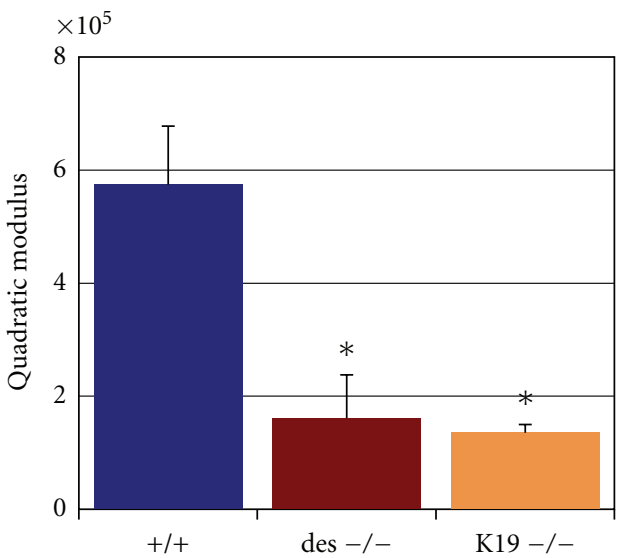

(b)

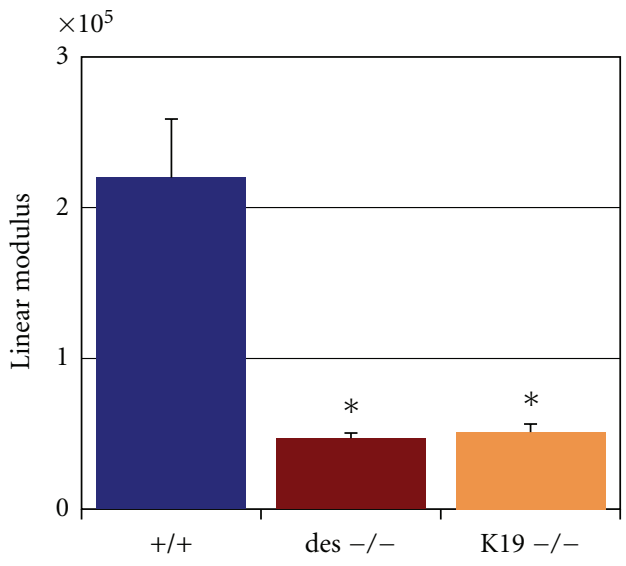

(d)

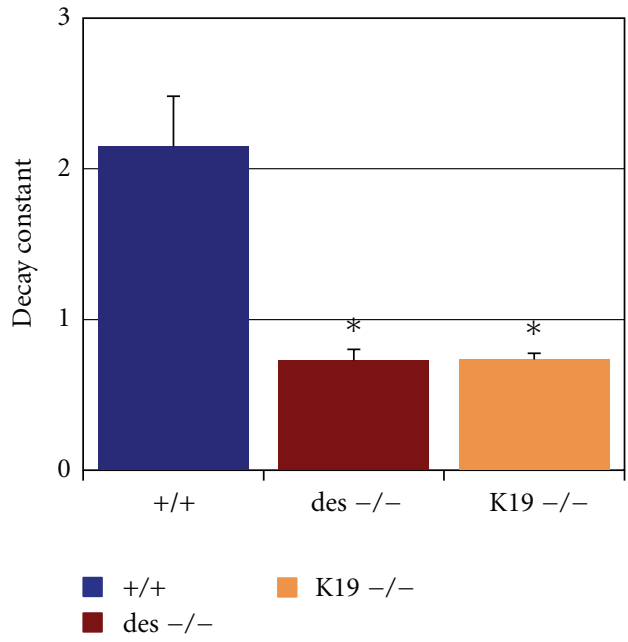

(f)

Figure 2: Mechanical response of fibers from young animals. Raw data (a, c, e) and regression coefficients (b, d, f) for (a-b) Cauchy stress-fiber strain (quadratic regression); (c-d) LaGrangian stress-fiber strain (linear regression); (e-f) radial strain-fiber strain (exponential regression) for fibers from 3-month-old wild-type (blue), desmin-null (red), and K19-null (orange) animals ( $n=5$ fibers/genotype). Different symbols within a color group represent data from individual experiments. Displayed regression lines in (a), (c), and (e) represent averaged coefficients for individual experiments reported in (b), (d), and (f). $*$ indicates significant difference from wild-type. $P$ values are reported in the text. 
TABLE 3: Summary of parameters that display significant post hoc differences $(P<0.05)$ between genotypes for fibers harvested from young mice. Raw data, means, and standard errors are provided in Figure 2. NS: no significant difference.

\begin{tabular}{lll}
\hline Genotype & des $-/-$ & $\mathrm{K} 19-/-$ \\
\hline$+/+$ & $\sigma_{\mathrm{LG}}, \sigma_{\mathrm{C}}, \varepsilon_{\mathrm{R}}, \nu$, & $\sigma_{\mathrm{LG}}, \sigma_{C}, \varepsilon_{\mathrm{R}}, \nu, V_{f}$ \\
Des $-/-$ & $V_{f}$ & $\mathrm{NS}$ \\
\hline
\end{tabular}

thus tighter coupling, between longitudinal and radial strains than desmin-null $(P<0.01)$ and K19-null $(P<0.01)$ fibers (Figure 1(f)).

This finding was consistent with our analysis of Poisson's ratio. As for fibers from older animals, regression revealed a power-law relationship between longitudinal and radial strains $\left(r^{2}>0.61\right)$, and Poisson's ratios for all genotypes far exceeded 0.5 , indicating considerable anisotropy. There were no significant differences in Poisson's ratio between desminnull and K19-null genotypes (Mean \pm SEM for des $-1-$ : $1.08 \pm 0.12$; ker $-/-: 1.19 \pm 0.08)$, but the Poisson's ratio of wild-type fibers $(2.43 \pm 0.33)$ was significantly higher than those of both desmin-null and K19-null fibers $(P<0.02)$. With respect to changes in volume in response to loading, as for older animals, all genotypes decreased in volume with increased deformation, and wild-type fibers displayed the most dramatic decrease in volume (Mean slope \pm SEM: $-1.08 \pm 0.19)$. This decrease was significantly greater $(P<$ 0.01 ) than that in both desmin-null and K19-null fibers (des $-/-: 0.24 \pm 0.05$; ker $-/-: 0.24 \pm 0.02$ ). Significant differences between parameters are summarized in Table 3 .

Though we have not yet compared the properties of fibers in 10-month-old to 3-month-old double-null mice, we confirmed differences in passive mechanical parameters between young and aged wild-type, desmin-null, and K19null mice using two-way ANOVA. Effects of genotype $(P<$ 0.0001 ), but no effect of age and no interaction were found on stress-strain and volume-strain relationships. However, a significant effect of genotype $(P<0.0001)$ and age $(P<$ $0.0001)$ and an interaction between genotype and age $(P<$ 0.0001 ) were observed for the coupling between radial strain and longitudinal strain as indicated by both exponential and power-law relationships. Post hoc analysis revealed a significant reduction in the exponential time constant and Poisson's ratio of longitudinal and radial strain for wild-type (exp: $P<0.008$; Poisson's ratio: $P<0.006$ ), desmin-null (exp: $P<0.005$; Poisson's ratio: $P<0.02$ ), and K19-null (exp: $P<0.001$; Poisson's ratio: $P<0.001$ ) fibers from aged mice compared to their younger counterparts (Figures $1(\mathrm{e})$ and $1(\mathrm{f})$ versus Figures 2(e) and 2(f)).

3.3. Structural Bases for Differences in Biomechanical Properties. In an effort to elucidate potential structural bases for differences in passive mechanical function between genotypes, we used phase-contrast microscopy to image the response of fibers from 10-month-old animals of each genotype at higher resolution (Figures 3-7). Wild-type fibers displayed well aligned Z-disks, even at high fiber strains
(Figure 4). In contrast, desmin-null fibers displayed the characteristic "basket-weave" pattern of misaligned myofibrils (Figure 5). Despite the misalignment of myofibrils in desmin-null mice, local sarcomere strain scaled consistently with fiber strain in both wild-type and desmin-null fibers (Figures 3(a), 3(b), 4, and 5). Additionally, membrane strain was also tightly correlated with sarcomere strain in both wild-type and desmin-null fibers, indicating continuity between the sarcolemma and myofibrillar lattice (Figures $3(\mathrm{c})$ and $3(\mathrm{~d})$ ).

In contrast, K19-null fibers appeared to have well aligned myofibrils, even in the presence of considerable shear (Figure 6). Though differences did not reach statistical significance due to the small sample size, there was considerable discontinuity between fiber strain and sarcomere strain for K19-null fibers compared to wild-type or desmin-null fibers (variability of each genotype in Figures 3(a) and 3(b); examples in Figures 4-6). Examination of the entire length of the fiber at multiple time points during experimentation suggested that this was not simply an artifact of fiber slippage at the knots or visible damage outside of the imaging window (Figures 6(b) and 6(c)). Additionally, similar to findings in desmin-null fibers, the absence of K19 did not appear to disrupt coupling between the sarcolemma and the contractile apparatus significantly, based on the strong correlation between local membrane strain and sarcomere strain (Figures 3(c) and 3(d)).

Double-null fibers, as expected, displayed phenotypes from both desmin-null and K19-null fibers. Visually, a lack of Z-disk alignment similar to that in desmin-null fibers was observed (Figure 7). As in K19-null fibers, however, there was also considerable discontinuity between fiber strain and sarcomere strain in double-null fibers (Figures 3(a), 3(b), and 7). As for all genotypes, there was no apparent decoupling between the sarcolemma and underlying myofibrils (Figures $3(\mathrm{c})$ and $3(\mathrm{~d})$ ).

\section{Discussion}

We characterized the mechanical response of skeletal muscle fibers from wild-type, desmin-null, K19-null, and desmin/ K19 double-null mice to passive tensile loading. Using a mechanical testing device that also enabled high-resolution imaging, we also characterized structural changes in fibers from each genotype during deformation. Our study extends previous work describing the roles of IFs in skeletal muscle morphology and function $[15,17-22,26]$ by showing that IFs comprised of desmin and of keratins each contribute to the biomechanical integrity of myofibrils in the sarcoplasm and their links to the sarcolemma. Our work also demonstrates that the weakening of these links in myofibers null for either of these proteins becomes more pronounced in older mice.

Experiments on wild-type and desmin-null fibers from 3-month-old and 10-month-old FVB mice confirmed and extended our previous characterization of fibers of these genotypes from 129/SVJ mice [26]. Reported regression coefficients and magnitudes for Cauchy stresses, LaGrangian 


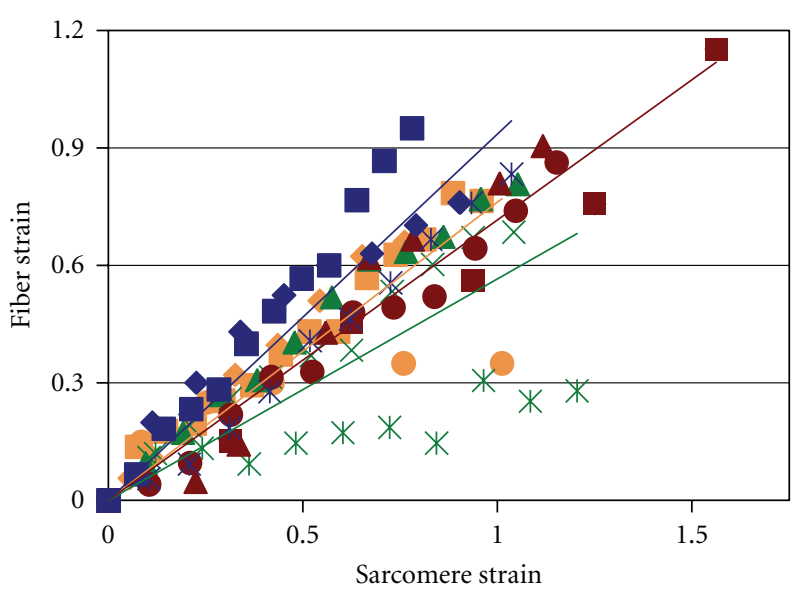

(a)

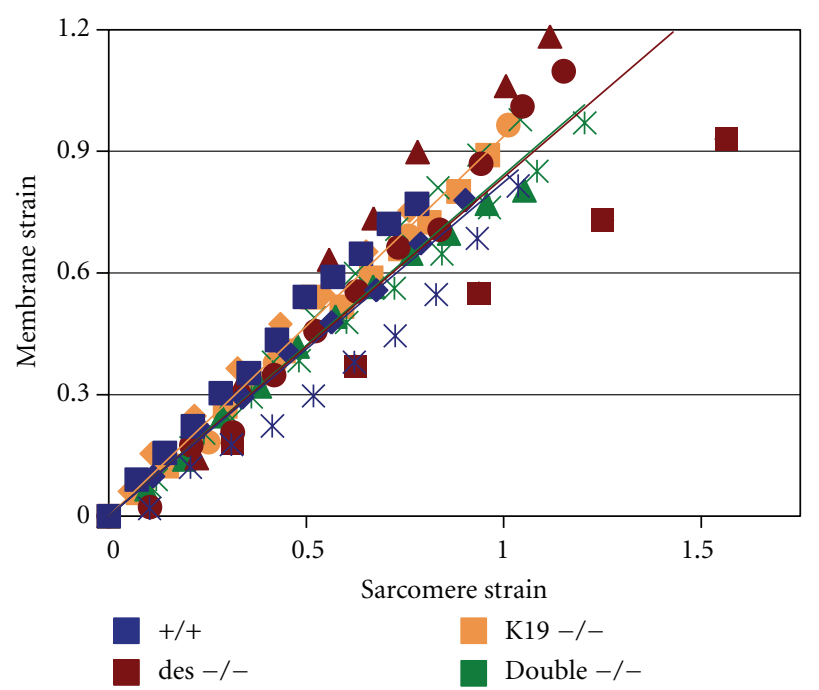

(c)

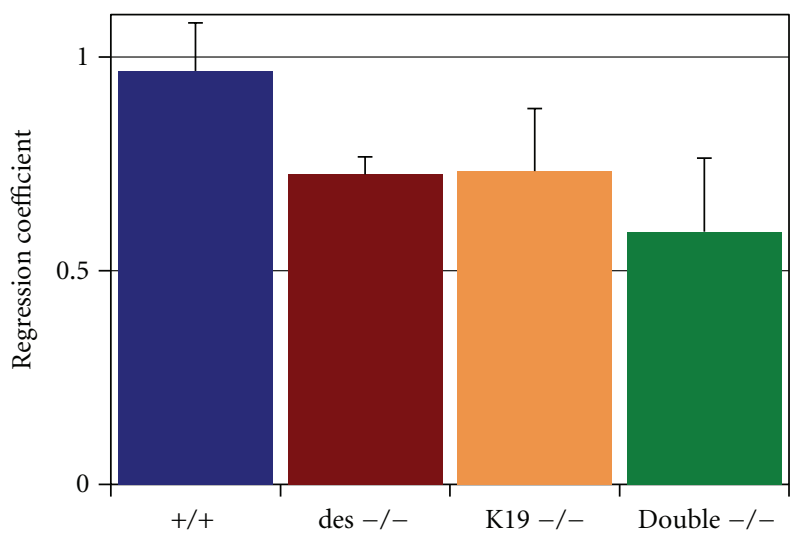

(b)

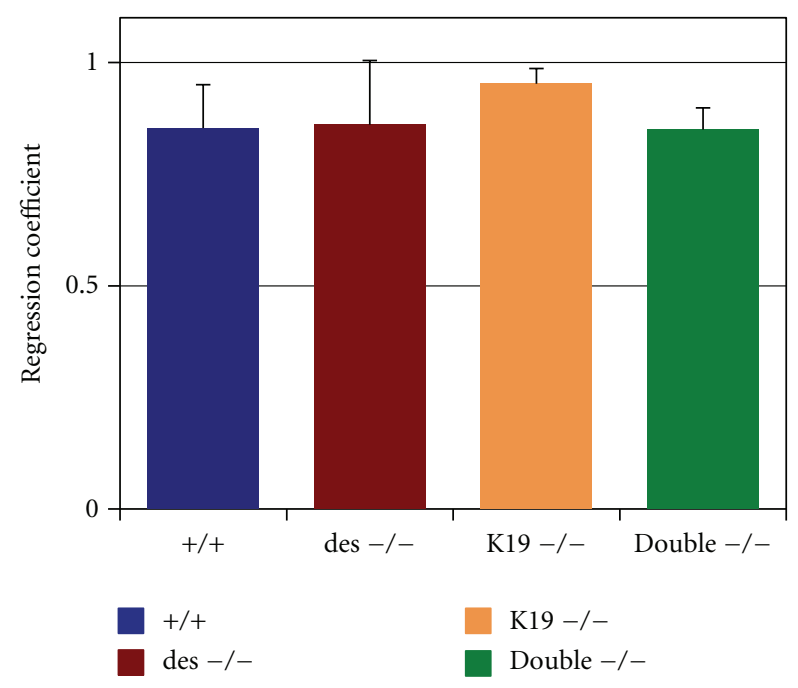

(d)

FIgURE 3: Relationships between global fiber strain and local membrane and sarcomere strain. Raw data (a, c) and regression coefficients (b, d) for (a-b) fiber strain-sarcomere strain; (c-d) membrane strain-sarcomere strain for fibers from 10-month-old wild-type (blue), desminnull (red), K19-null (orange), and double-null (green) animals ( $n=3$ fibers/genotype). Different symbols within a color group represent data from individual experiments. Displayed regression lines in (a) and (c) represent averaged coefficients for individual experiments reported in (b) and (d).

stresses, and radial strains are consistent with those previously reported, suggesting that the murine strain does not influence passive mechanical properties in skeletal muscle. Differences in elastic moduli between wild-type and desminnull fibers, reported for both LaGrangian and Cauchy stress-strain curves, indicate a role for desmin in both defining inherent load-bearing capabilities within the muscle fiber and regulating changes in fiber geometry that further influence force transmission (Figures $1(\mathrm{a})-1(\mathrm{~d})$, and $2(\mathrm{a})-$ $2(\mathrm{~d})$ ). The latter finding is also consistent with significantly decreased coupling between longitudinal and radial strains and reduced changes in volume following loading in desminnull fibers compared to wild-type (Figures 1(e), 1(f), 2(e), and 2(f), Poisson's ratios).
Given the misalignment of Z-disks observed in single fibers and in whole muscle (Figure 5; $[15,16,18,26]$ ), it is likely that a major site of desmin's influence on force transmission is within the myofibrillar lattice. Within this region, desmin enhances the effectiveness in transmission of shear loads and coordinates tensile load-bearing between myofibrils. However, it remains an open question whether desmin also influences force transmission at the sarcolemma. On one hand, this study reports a strong correlation of membrane strain and sarcomere strain (Figures 3(c) and $3(d)$ ). This would suggest limited influence of desmin at the sarcolemma, consistent with the tight coupling between Zdisks and costameric proteins observed in single desmin-null EDL fibers [26], and intact costameres in quadriceps muscle 


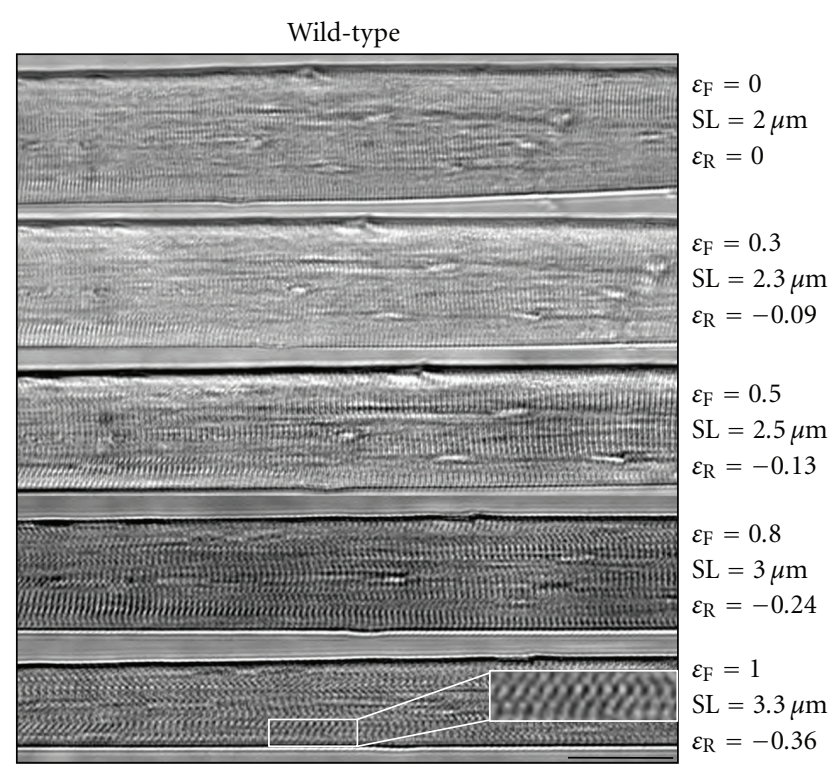

Figure 4: Geometric and structural response of a 10-month-old wild-type fiber to tensile loading. The same region of the fiber, imaged using phase-contrast microscopy, is shown at five different deformations. Fiber strains, radial strains, and sarcomere lengths are reported to the right of the fiber for each deformation. The fiber displays well aligned Z-lines (dark vertical stripes), consistently increasing sarcomere strain, and decreasing fiber diameter with increased fiber deformation. A sample region of the fiber that displays particularly well aligned Z-lines that are experiencing some shear, inferred through the slanting of Z-lines, is marked with a white box. The boxed region has been computationally enlarged $2 \mathrm{x}$ for clarity. At high strains $\left(\varepsilon_{\mathrm{F}}>0.8\right)$, artifactual thickening of Zlines occurs due to shear-induced offsets of $Z$-lines in deeper regions of the fiber. Bar $=50 \mu \mathrm{m}$.

from desmin-null mice [15]. On the other hand, significant costamere disruption is seen in tibialis anterior and EDL muscle [15], suggesting that desmin does, in fact, influence costamere, and thus membrane, coupling to the underlying myofibrillar lattice.

Examination of K19-null fibers from three-month-old FVB mice indicated, for the first time, a role for keratins in regulating the passive mechanical properties of muscle. Similar to desmin-null fibers, K19-null fibers are more compliant than wild-type fibers, based on LaGrangian and Cauchy stress-strain curves (Figures $1(\mathrm{a})-1(\mathrm{~d})$, and 2(a)$2(d)$ ). In addition, K19-null fibers from younger mice exhibit poor coupling between longitudinal and radial strain (Figures 2(e) and 2(f), Poisson's ratios). Interestingly, coupling between longitudinal and radial strain, and thus perhaps the efficiency of lateral force transmission, decreases for all genotypes with aging (Figures 1(e), 1(f), 2(e), and 2(f), Poisson's ratios). This may be a result of myofibril loss and a consequent increase in intramyofibrillar space $[28,29]$. In contrast to desmin-null fibers, though, coupling between longitudinal and radial strain was not significantly decreased in 10-month-old K19-null fibers compared to wild-type fibers (Figures 1(e) and 1(f)), and Z-disks appeared to

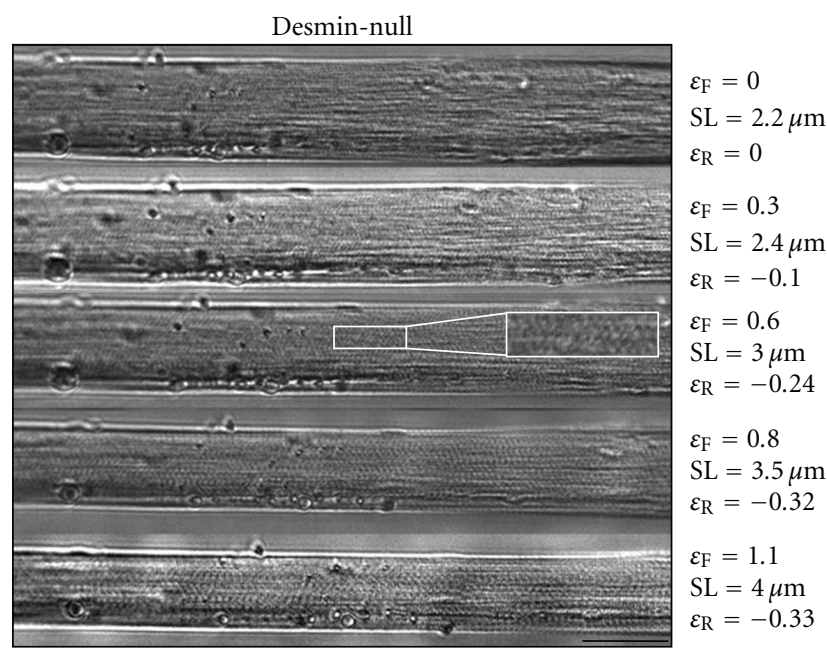

Figure 5: Geometric and structural response of a 10-monthold desmin-null fiber to tensile loading. The same region of the fiber, imaged using phase-contrast microscopy, is shown at five different deformations. Fiber strains, radial strains, and sarcomere lengths are reported to the right of the fiber for each deformation. The fiber displays misaligned Z-lines, but consistently increasing sarcomere strain and decreasing fiber diameter with increased fiber deformation with increased fiber deformation. Misalignment is characterized by a striking basketweave pattern where no clear Zline orientation may be inferred. A sample region of the fiber that displays this pattern is marked with a white box, and may be compared to the well aligned region shown in Figure 4. The boxed region has been computationally enlarged $2 \mathrm{x}$ for clarity. Desmin-null fibers display dramatically more debris on their external membrane compared to wild-type fibers. This observation is consistent with the increased "stickiness" experienced during fiber dissection, possibly resulting from differences in extracellular matrix composition. Bar $=50 \mu \mathrm{m}$.

remain well aligned, suggesting different mechanisms by which K19 and desmin influence passive biomechanical properties. Taken together, these results raise the possibility that genotypic differences in longitudinal and radial coupling may in part be a result of differences in Z-disk connectivity, both longitudinally and laterally $[16,18,22,26]$.

Also unlike desmin-null fibers, K19-null fibers reveal a poor correlation between fiber strain and sarcomere strain (Figures 3(a), 3(b), 5, and 6). The reason for this lack of correlation remains unclear. Based on disrupted costameres and the dramatic accumulation of mitochondria in some subsarcolemmal regions of K19-null muscle [22], decoupling between the membrane and myofibrillar response to deformation was predicted; however, coupling between the plasma membrane and the contractile apparatus does not appear to be dramatically disrupted, at least over a length scale of 100-200 $\mu \mathrm{m}$ (Figures 3(c) and 3(d)). There are at least two reasonable explanations for this paradox, which are also applicable to understanding desmin-mediated connectivity at the costameres. First, when disrupted costameres have been observed in whole muscle sections $[15,22]$, there appear to be at least a few costameres in a given fiber that are intact. This limited connectivity may be sufficient to 


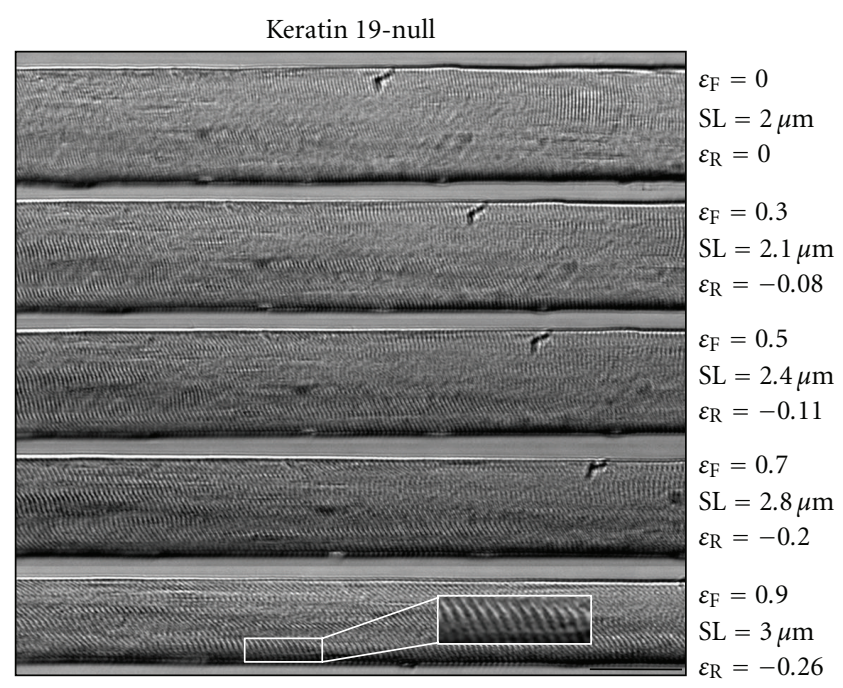

(a)

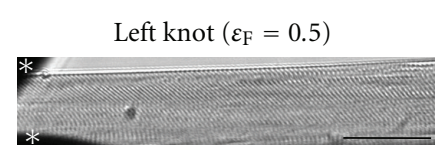

(b)

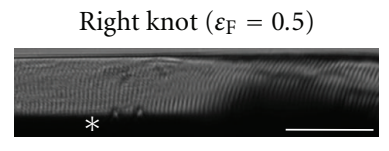

(c)
Figure 6: Geometric and structural response of a 10-month-old K19-null fiber to tensile loading. (a) The same region of the fiber, imaged using phase-contrast microscopy, is shown at five different deformations. Fiber strains, radial strains, and sarcomere lengths are reported to the right of the fiber for each deformation. The fiber displays aligned Z-lines, but discontinuity between fiber deformation and sarcomere strain. This fiber also displays considerable shear, as seen by the slanting of Z-lines (dark lines). A sample region of the fiber that displays particularly well aligned $\mathrm{Z}$-lines that are experiencing some shear is marked with a white box. The boxed region has been computationally enlarged $2 \mathrm{x}$ for clarity. There is no slippage or damage of the fiber at the (b) left edge or (c) right edge, imaged in the vicinity of the knot and pins (starred black regions in the periphery of the image) at a moderate fiber strain of 0.5 . This may be concluded from the gradual reduction in of the slant of Z-disks away from the knot, which is located to the left in (b) and to the right in (c). Bar $=50 \mu \mathrm{m}$.

tether the membrane to the underlying cytoskeleton, though not necessarily strongly or effectively. The fraction of intact costameres is likely to be even higher in successfully teased (i.e., more robust) single fibers from IF-null muscles (this study, [26]), further enhancing apparent connectivity. The second explanation arises from the fact that multiple structural proteins, including other intermediate filaments such as K8, localize to costameres and thus likely contribute to the mechanical properties of the connection between individual costameres and underlying Z-disks (cf. [30, 31]). In this case, costameres may still be weakly coupled to myofibrils via remaining or compensatory structural proteins. Examination of more subtle differences in connectivity at the scale of individual or a few sarcomeres under conditions of radial tensile loading (e.g., [30]) may reveal differences in

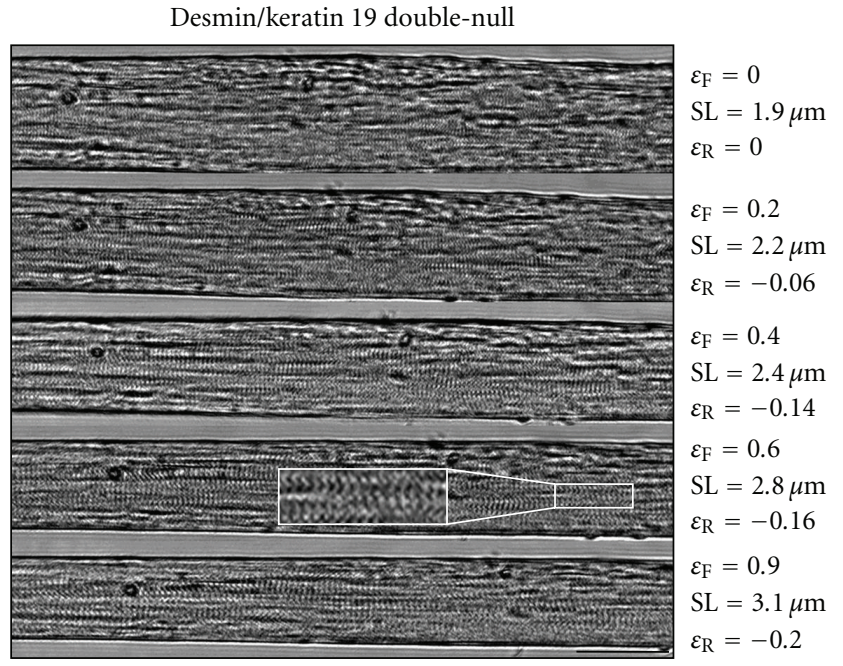

Figure 7: Geometric and structural response of a 10-monthold double-null fiber to tensile loading. The same region of the fiber, imaged using phase-contrast microscopy, is shown at five different deformations. Fiber strains, radial strains, and sarcomere lengths are reported to the right of the fiber for each deformation. Fiber displays both misaligned Z-lines as well as discontinuity between fiber, sarcomere, and radial strains. A sample region of the fiber that displays the basketweave pattern characteristic of misalignment is marked with a white box. The boxed region has been also computationally enlarged $2 \mathrm{x}$ for clarity. As for desminnull fibers, double-null fibers display dramatically more debris on their external membrane compared to wild-type fibers. Bar = $50 \mu \mathrm{m}$.

local compliance that could then underlie differences in the efficiency of lateral force transmission.

Additional insight into the different roles of desmin and keratin emerged from the examination of desmin/K19 double-null fibers in 10-month-old mice. The similar fiber compliance of double-null fibers and desmin-null fibers, but increased compliance compared to K19-null fibers (Figures $1(\mathrm{a})-1(\mathrm{~d})$ ) parallels decreased myofibrillar organization within desmin-null and double-null fibers compared to K19null fibers (Figures 5-7). Our findings in single myofibers are also consistent with biomechanical results in whole muscle, where deficits in specific tension are similar and more severe in desmin-null and double-null tibialis anterior muscle than in K19-null muscle [18]. These data support the hypothesis that myofibrils must be engaged and coordinated to maximally support mechanical loads. It will be interesting to continue the characterization of double-null fibers in younger mice, where phenotypic differences between desmin-null and K19-null fibers were not significant, to address developmental differences in IF function.

Collectively, integration of mechanical and structural data from this work and previous studies support a model in which membrane-myofibril connectivity, guided by two sets of IFs, composed of keratins and of desmin, dictates the radial transmission of tensile and shear loads into and out of the cell. In an outside-in model, this connectivity would enable the recruitment of longitudinal load-bearing 
structures, predominantly coordinated by desmin, within the myofibrillar lattice. Conversely, in an inside-out model, connectivity between myofibrils and the membrane would enable the reliable transmission of myofibrillar forces, again coordinated by desmin, to the extracellular matrix.

Finally, this work illustrates the importance of studying single muscle fibers to the interpretation of biomechanical data from knockout models. Increased passive compliance measured in desmin-null fibers (Figures 1 and 2; [26]) is contrary to the increased passive stiffness reported in desmin-null muscles $[17,32]$. These differences are most likely due to secondary remodeling of extracellular matrix, though this remains to be tested directly. Given the increasing relevance of extracellular matrix remodeling to our understanding of myopathies and muscular dystrophies [2325], comparative studies between single fibers and whole muscle or fiber bundles may reveal interesting and important details regarding roles of intermediate filaments in initiating physiologically relevant extracellular signaling pathways.

\section{Conclusions}

We have characterized the structural and mechanical response of single fibers from wild-type, desmin-null, K19-null, and desmin/K19 double-null mice to passive tensile loading. Through this work, we provide the first direct evidence, in an experimental system free from influences of the extracellular matrix, that desmin and keratin 19 differentially influence passive force transmission in single muscle fibers. Our work has strong implications for our understanding of the role of intermediate filaments on structure-function relationships in skeletal muscle. In addition, our work is important for interpreting structural and biomechanical changes resulting from desminopathies and other myopathies linked to intermediate filaments and their associated proteins [33-37].

\section{Acknowledgments}

The authors acknowledge contributions to device modification by Jiemin Wu. This research was supported by grant 1RO1AR055928 to RJB and SBS, and K01AR053235 and 1R01AR059179 to RML, from NIAMS/NIH, and grant 69236 to RJB from the Muscular Dystrophy Association.

\section{References}

[1] R. J. Monti, R. R. Roy, J. A. Hodgson, and V. R. Edgerton, "Transmission of forces within mammalian skeletal muscles," Journal of Biomechanics, vol. 32, no. 4, pp. 371-380, 1999.

[2] J. G. Tidball, "Force transmission across muscle cell membranes," Journal of Biomechanics, vol. 24, supplement 1, pp. 43-52, 1991.

[3] R. J. Bloch and H. Gonzalez-Serratos, "Lateral force transmission across costameres in skeletal muscle," Exercise and Sport Sciences Reviews, vol. 31, no. 2, pp. 73-78, 2003.

[4] S. F. Street, "Lateral transmission of tension in frog myofibers: a myofibrillar network and transverse cytoskeletal connections are possible transmitters," Journal of Cellular Physiology, vol. 114, no. 3, pp. 346-364, 1983.
[5] R. J. Bloch, Y. Capetanaki, A. O’Neill et al., "Costameres: repeating structures at the sarcolemma of skeletal muscle," Clinical Orthopaedics and Related Research, no. 403, pp. S203S210, 2002.

[6] Y. Capetanaki, R. J. Bloch, A. Kouloumenta, M. Mavroidis, and S. Psarras, "Muscle intermediate filaments and their links to membranes and membranous organelles," Experimental Cell Research, vol. 313, no. 10, pp. 2063-2076, 2007.

[7] D. Paulin and Z. Li, "Desmin: a major intermediate filament protein essential for the structural integrity and function of muscle," Experimental Cell Research, vol. 301, no. 1, pp. 1-7, 2004.

[8] B. L. Granger and E. Lazarides, "Desmin and vimentin coexist at the periphery of the myofibril $\mathrm{Z}$ disc," Cell, vol. 18, no. 4, pp. 1053-1063, 1979.

[9] F. L. Richardson, M. H. Stromer, T. W. Huiatt, and R. M. Robson, "Immunoelectron and immunofluorescence localization of desmin in mature avian muscles," European Journal of Cell Biology, vol. 26, no. 1, pp. 91-101, 1981.

[10] K. T. Tokuyasu, A. H. Dutton, and S. J. Singer, "Immunoelectron microscopic studies of desmin (skeletin) localization and intermediate filament organization in chicken skeletal muscle," Journal of Cell Biology, vol. 96, no. 6, pp. 1727-1735, 1983.

[11] K. Wang and R. Ramirez-Mitchell, "A network of transverse and longitudinal intermediate filaments is associated with sarcomeres of adult vertebrate skeletal muscle," Journal of Cell Biology, vol. 96, no. 2, pp. 562-570, 1983.

[12] J. V. Pardo, J. D. Siliciano, and S. W. Craig, "Vinculin is a component of an extensive network of myofibril-sarcolemma attachment regions in cardiac muscle fibers," Journal of Cell Biology, vol. 97, no. 4, pp. 1081-1088, 1983.

[13] J. V. Pardo, J. D. Siliciano, and S. W. Craig, "A vinculincontaining cortical lattice in skeletal muscle: transverse lattice elements ("costameres") mark sites of attachment between myofibrils and sarcolemma," Proceedings of the National Academy of Sciences of the United States of America, vol. 80, no. 4, pp. 1008-1012, 1983.

[14] D. J. Milner, M. Mavroidis, N. Weisleder, and Y. Capetanaki, "Desmin cytoskeleton linked to muscle mitochondrial distribution and respiratory function," Journal of Cell Biology, vol. 150, no. 6, pp. 1283-1297, 2000.

[15] A. O’Neill, M. W. Williams, W. G. Resneck, D. J. Milner, Y. Capetanaki, and R. J. Bloch, "Sarcolemmal organization in skeletal muscle lacking desmin: evidence for cytokeratins associated with the membrane skeleton at costameres," Molecular Biology of the Cell, vol. 13, no. 7, pp. 2347-2359, 2002.

[16] S. B. Shah, F. C. Su, K. Jordan et al., "Evidence for increased myofibrillar mobility in desmin-null mouse skeletal muscle," Journal of Experimental Biology, vol. 205, part 3, pp. 321-325, 2002.

[17] M. Sam, S. Shah, J. Fridén, D. J. Milner, Y. Capetanaki, and R. L. Lieber, "Desmin knockout muscles generate lower stress and are less vulnerable to injury compared with wild-type muscles," American Journal of Physiology-Cell Physiology, vol. 279, no. 4, pp. C1116-C1122, 2000.

[18] R. M. Lovering, A. O’Neill, J. M. Muriel, B. L. Prosser, J. Strong, and R. J. Bloch, "Physiology, structure, and susceptibility to injury of skeletal muscle in mice lacking keratin 19-based and desmin-based intermediate filaments," American Journal of Physiology - Cell Physiology, vol. 300, no. 4, pp. C803-C813, 2011. 
[19] A. M. Boriek, Y. Capetanaki, W. Hwang et al., "Desmin integrates the three-dimensional mechanical properties of muscles," American Journal of Physiology —Cell Physiology, vol. 280, no. 1, pp. C46-C52, 2001.

[20] M. R. Stone, A. O’Neill, D. Catino, and R. J. Bloch, “Specific interaction of the actin-binding domain of dystrophin with intermediate filaments containing keratin 19," Molecular Biology of the Cell, vol. 16, no. 9, pp. 4280-4293, 2005.

[21] J. A. Ursitti, P. C. Lee, W. G. Resneck et al., "Cloning and characterization of cytokeratins 8 and 19 in adult rat striated muscle: interaction with the dystrophin glycoprotein complex," Journal of Biological Chemistry, vol. 279, no. 40, pp. 41830-41838, 2004.

[22] M. R. Stone, A. O’Neill, R. M. Lovering et al., "Absence of keratin 19 in mice causes skeletal myopathy with mitochondrial and sarcolemmal reorganization," Journal of Cell Science, vol. 120, part 22, pp. 3999-4008, 2007.

[23] J. M. Boer, E. J. de Meijer, E. M. Mank, G. B. van Ommen, and J. T. den Dunnen, "Expression profiling in stably regenerating skeletal muscle of dystrophin-deficient mdx mice," Neuromuscular Disorders, vol. 12, supplement 1, pp. S118-S124, 2002.

[24] M. Marotta, C. Ruiz-Roig, Y. Sarria et al., "Muscle genomewide expression profiling during disease evolution in $\mathrm{mdx}$ mice," Physiological Genomics, vol. 37, no. 2, pp. 119-132, 2009.

[25] G. A. Meyer and R. L. Lieber, "Elucidation of extracellular matrix mechanics from muscle fibers and fiber bundles," Journal of Biomechanics, vol. 44, no. 4, pp. 771-773, 2011.

[26] S. B. Shah, J. Davis, N. Weisleder et al., "Structural and functional roles of desmin in mouse skeletal muscle during passive deformation," Biophysical Journal, vol. 86, no. 5, pp. 2993-3008, 2004.

[27] S. B. Shah and R. L. Lieber, "Simultaneous imaging and functional assessment of cytoskeletal protein connections in passively loaded single muscle cells," Journal of Histochemistry and Cytochemistry, vol. 51, no. 1, pp. 19-29, 2003.

[28] S. Bensamoun, L. Stevens, M. J. Fleury, G. Bellon, F. Goubel, and M. C. H. B. Tho, "Macroscopic-microscopic characterization of the passive mechanical properties in rat soleus muscle," Journal of Biomechanics, vol. 39, no. 3, pp. 568-578, 2006.

[29] T. Ansved and L. Edstrom, "Effects of age on fibre structure, ultrastructure and expression of desmin and spectrin in fastand slow-twitch rat muscles," Journal of Anatomy, vol. 174, pp. 61-79, 1991.

[30] K. P. García-Pelagio, R. J. Bloch, A. Ortega, and H. GonzálezSerratos, "Biomechanics of the sarcolemma and costameres in single skeletal muscle fibers from normal and dystrophin-null mice," Journal of Muscle Research and Cell Motility, vol. 31, no. 5-6, pp. 323-336, 2011.

[31] G. A. Meyer, B. Kiss, S. R. Ward, D. L. Morgan, M. S. Z. Kellermayer, and R. L. Lieber, "Theoretical predictions of the effects of force transmission by desmin on intersarcomere dynamics," Biophysical Journal, vol. 98, no. 2, pp. 258-266, 2010.

[32] J. Anderson, Z. Li, and F. Goubel, "Passive stiffness is increased in soleus muscle of desmin knockout mouse," Muscle and Nerve, vol. 24, no. 8, pp. 1090-1092, 2001.

[33] B. L. Banwell, "Intermediate filament-related myopathies," Pediatric Neurology, vol. 24, no. 4, pp. 257-263, 2001.

[34] K. Nowak, K. McCullagh, E. Poon, and K. E. Davies, "Muscular dystrophies related to the cytoskeleton/nuclear envelope," Novartis Foundation Symposium, vol. 264, pp. 98-111, 2005.
[35] D. Paulin, A. Huet, L. Khanamyrian, and Z. Xue, "Desminopathies in muscle disease," Journal of Pathology, vol. 204, no. 4, pp. 418-427, 2004.

[36] D. J. Blake and E. Martin-Rendon, "Intermediate filaments and the function of the dystrophin-protein complex," Trends in Cardiovascular Medicine, vol. 12, no. 5, pp. 224-228, 2002.

[37] H. H. Goebel and I. A. P. Warlo, "Progress in desmin-related myopathies," Journal of Child Neurology, vol. 15, no. 9, pp. 565-572, 2000. 

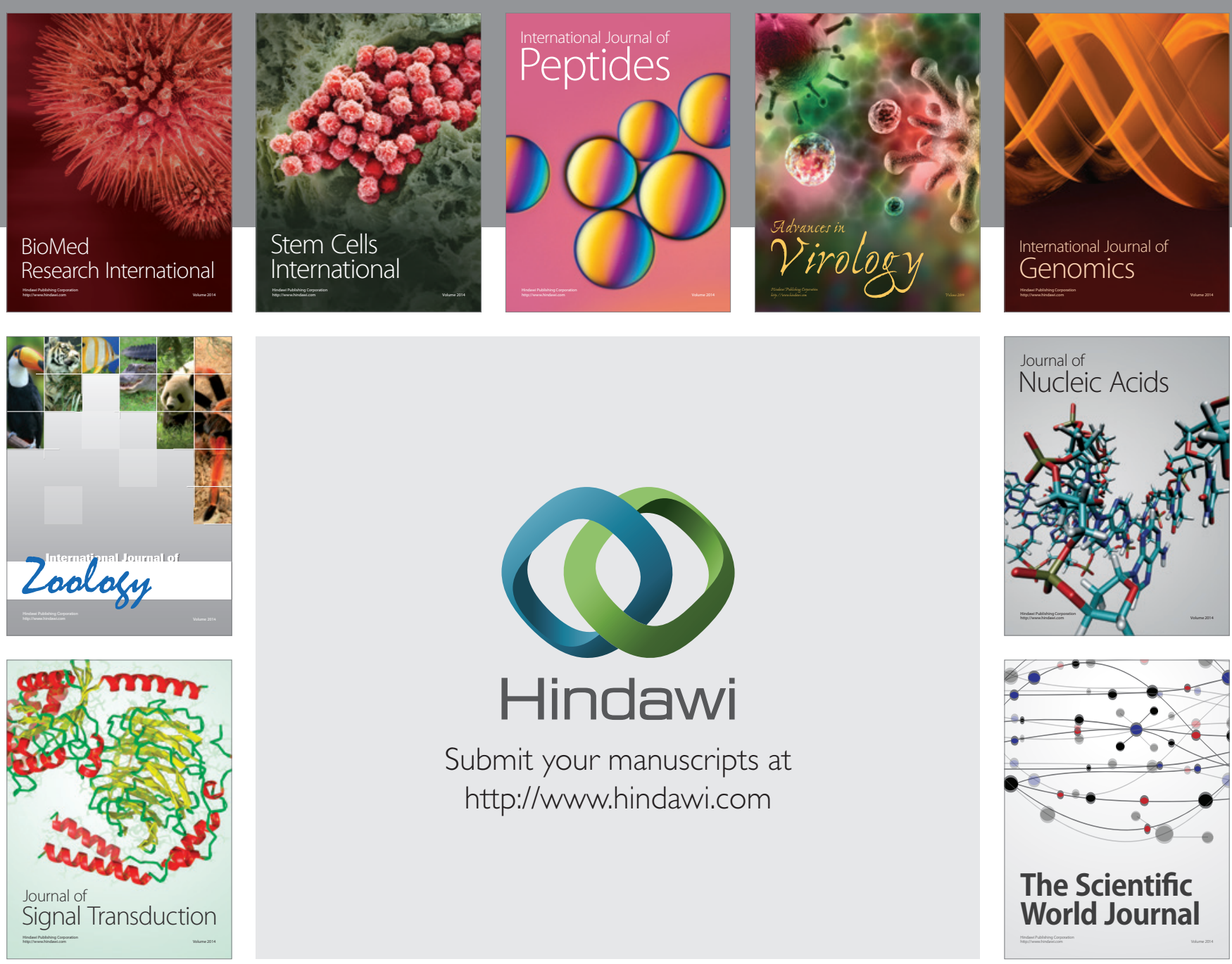

Submit your manuscripts at

http://www.hindawi.com
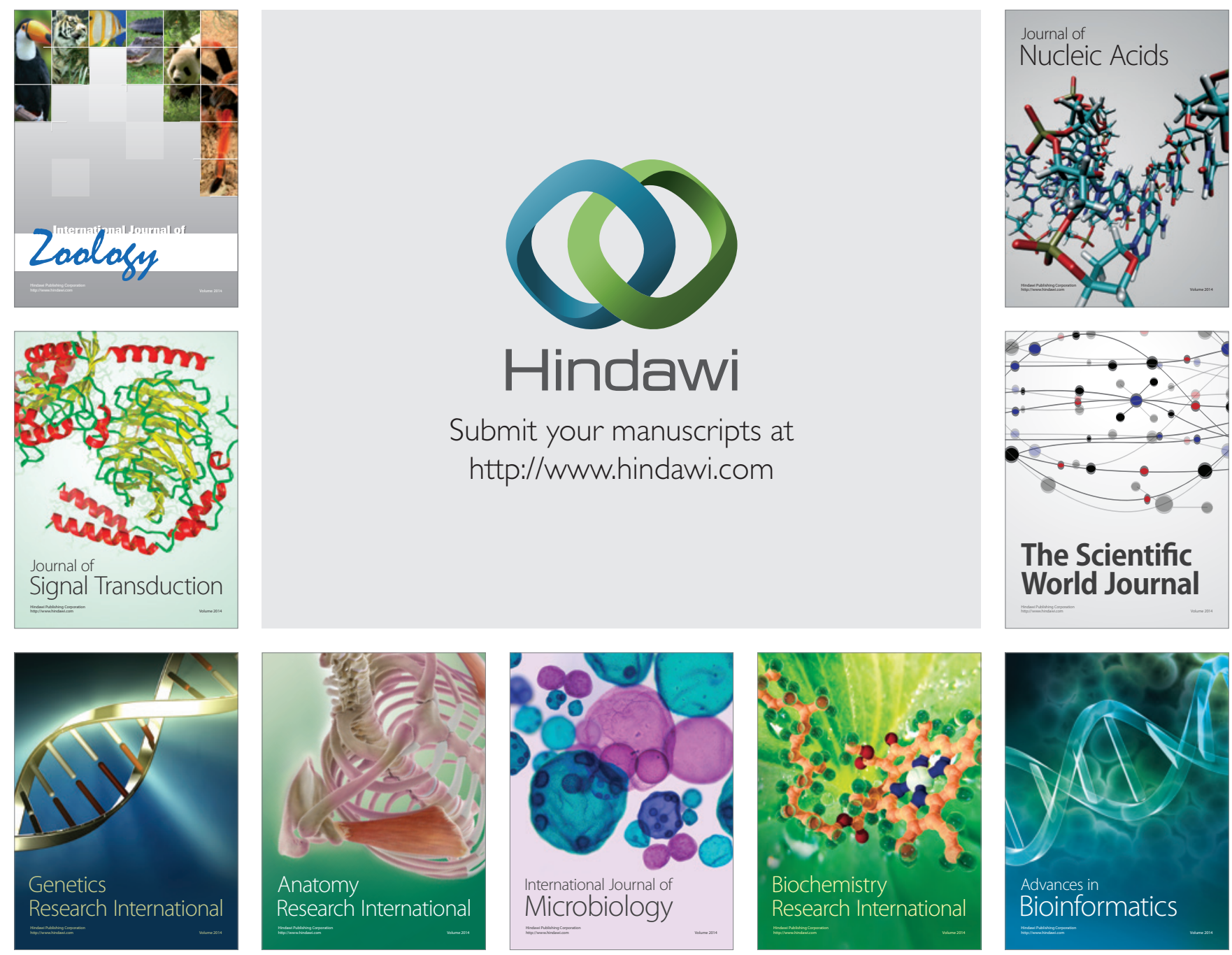

The Scientific World Journal
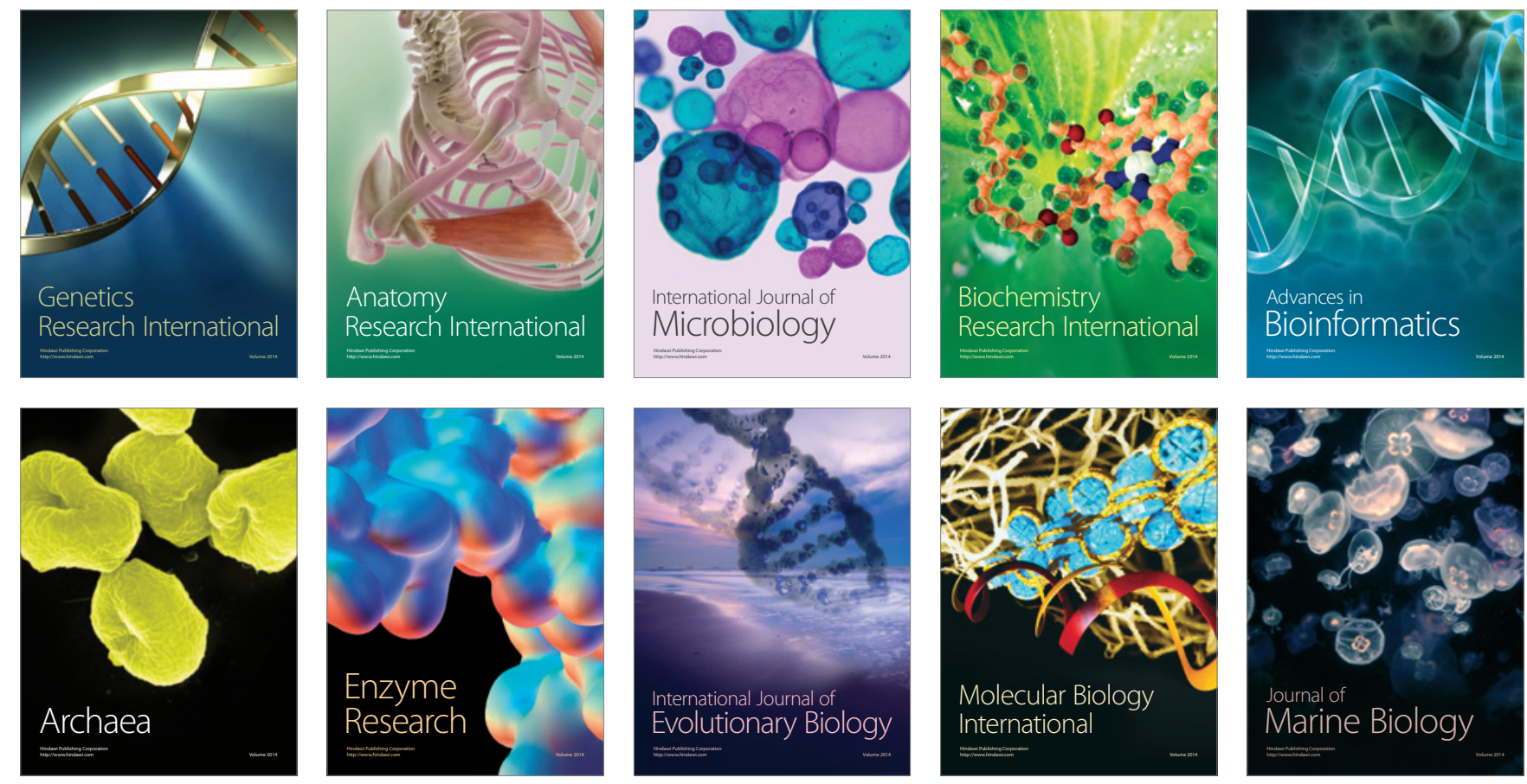